\title{
Ultrasensitive Detection of Water Contaminants, Biomarkers and illegal Drugs Using Active 3D Metallic Nanostructures
}

\author{
Carlos Escobedo \\ Department of Chemical Engineering, \\ Queen's University, Canada
}

Three-dimensional metallic nanostructures produced via laser interference support the generation of surface plasmon polaritons. This type of nanostructures are well-suited for pointof-use (bio)sensing applications, are extremely costeffective, and can be easily fabricated on practically any flat surface. Here, we present a new generation of metallic nanostructures fabricated using holographic laser-inscription that are capable of producing accurate photonic signals that can be employed as label-free (bio)sensors for the rapid, in situ detection and identification of biomarkers of diseases, illegal drugs, water contaminants and terrorism agents. These biosensing platforms consist of a network of threedimensional metallic nanostructures with thickness in the order of tens of nanometers, which can be employed for both surface plasmon resonance (SPR) and surface-enhanced Raman scattering (SERS). The platform utilize smartphoneanalogous, off-the-shelf inexpensive optical components for the generation and detection of the photonic signal. We demonstrate sensing of solutions with different refractive indices and real-time detection of biologically relevant analytes including proteins, water contaminants, illicit drugs and pathogenic bacteria with a sensitivity of $\sim 103$ PIU/RIU. This work presents a significant advancement towards the development of fully-integrated, handheld portable (bio)sensing platform for point-of-use applications requiring (bio)detection in real-time. 